\title{
Why it is impossible to recover periodontal disease areas to the pre-disease stage
}

\author{
José Ricardo Kina* \\ Department of Surgery and Integrated Clinic, Dental School of Araçatuba, São Paulo State University, Brazil
}

To prevent or to treat any disease always will be necessary to eliminate or to establish control in all etiologic factors or/and improve host local defence or/and general resistance against the entire etiologic factors, to promote homeostasis in diseased areas through a longstated period. However, the treatment of the periodontal disease sequel includes surgical exposure of the area, debridement of the diseased site and the adjacent involved root surface, complementary root physical and chemical treatment, and subsequently the application of respective or regenerative procedures, via altering the periodontal bony defect morphology and then surgical closure of the area. These procedures generally are effective in controlling the periodontal contamination by bacteria and their products but are unpredictable to recuperate periodontal destructed tissue at pre-disease stage. In the early stages of periodontal wound healing, the wound stabilization and nutrition may be a critical variable to achieve regeneration. All healing wounds proceed through into three phases during the process of healing, following injury: inflammation, fibroblastic-granulation, and matrix formation and remodelling. In the sequencing of these events, during the early phase of healing, a fibrin clot is formed. In any wounds, this fibrin clot bridges the space between two vascular wound margins and serves as a base that epithelial cells migrate across to cover the wound, inducing protection to the underlying connective tissue as healing progresses. Periodontal surgical wounds follow a similar healing pattern, but there are some significant differences in this specific wound healing environment that may affect the outcome of the procedures. When periodontal wounds are closed and sutured, one of the wound margins is an avascular and rigid periodontitis-affected and altered root surface. This detail induces a fibrin clot formation with a fragile initial attachment to the altered root surface, to prevent epithelial down growth and to form a scaffold for development of a cell and collagen fibre attachment mechanism. Then a fibrin clot adherent to the altered root surface is a fragile but vital part of early periodontal wound healing. The fibrin clot must form and adhere to the altered root surface for adequate time to allow for proper wound maturation, including connective tissue formation and development, before a new connective tissue attachment can occur. If this first series of events is disrupted, or if the initial attachment of fibrin or/and immature connective tissue is ruptured, then a pattern of healing including a long junctional epithelium to the base of the original periodontal pocket is expected to occur. In periodontal surgery procedure the early wound healing stability is easily disturbed inducing a disruption in the fibrin clot, which is frail attached to the altered root surface. This occurrence allows in this unique healing site a communication between the underlying connective tissue and the contaminated, humid and warm oral environment as healing progresses. To prevent infection, epithelial proliferations extend apically on the tooth aspect, establishing a long junctional epithelium attached to the root surface by hemidesmosomes. The long junctional epithelium is a fragile structural and functional adaptation enabled to produce a defensive biological mechanism, which is responsible to control the constant microbial challenge by isolating the exposed connective tissue in the inner surface of the wound from contaminated oral environment. However, this fragile protective structure also effectively prevents connective tissue from gaining access to the root surface and precludes periodontal regeneration to the predisease stage. As well as in periodontal disease, dentoalveolar abscess occur in periodontal apical tissues, when polymicrobial opportunistic pathogens resembling periodontopathogen bacterial flora, infect the dental pulp assisted by a predisposing factor as dental caries. The infection induces pulper necrosis, which extends through the root canal system of the tooth, progressing rapidly to involve the periodontal periapical structures. The development of infection in the periapical region, defines a localized area of inflammation arising in the periodontal ligament space, implicating in periodontal tissue contamination and destruction, similar as induced by periodontal disease. However, when an endodontic therapy is done properly, the pathway that leads the periapical area to the contaminated oral cavity is obstructed. Without junctional epithelium interference, the periodontal periapical tissues as cementum, bone and periodontal ligament may regenerate, acquiring pre-disease condition, even without any specific physical and chemical treatment on the periodontal periapical contaminated root surface. Then the major factors inhibiting periodontal disease tissues to achieve predictable regeneration appears to be: 1- the nature and complexity of marginal periodontal tissue around the teeth, the unique tissue in the body, structured into the basic components of cementum, alveolar bone and periodontal and gingival ligament, which are separated from an infected, humid and warm oral environment by a fragile junctional epithelium.

\section{References}

1. Kina JR, Kina J, Kina EF, Kina M, Soubhia AM (2008) Presence of bacteria in dentinal tubules. J Appl Oral Sci 16: 205-258. [Crossref]

2. Kina JR, Suzuki TY, Kina J, Kina M, Kina EF (2013) Reparative phase events on periodontal disease progression: interpretation and considerations. Int J Microbiol Res 5: 439-444.

Correspondence to: José Ricardo Kina, Division of Periodontics, Department of Surgery and Integrated Clinic, Dental School of Araçatuba, São Paulo State University, Brazil, Tel: +55-18-3636-3200; E-mail: kinajr@hotmail.com

Received: November 13, 2017; Accepted: December 11, 2017; Published: December 14, 2017 
3. Kina JR, Suzuki TY, Kina J, Kina M, Kina EF (2016) Non-Inflammatory Destructive Periodontal Disease. J Appl Oral Sci 10: 50-57. [Crossref]
4. Polson AM (1994) Periodontal regeneration: current status and directions. Chicago: Quintessence.

Copyright: (O2017 Kina JR. This is an open-access article distributed under the terms of the Creative Commons Attribution License, which permits unrestricted use, distribution, and reproduction in any medium, provided the original author and source are credited. 